\title{
Assessment of MRI-Detected Breast Lesions: A Benign Correlate on Second-Look Ultrasound Can Safely Exclude Malignancy
}

\author{
Karin Hellerhoff ${ }^{a}$ Hanna Dietrich ${ }^{b}$ Regina Schinner ${ }^{b}$ \\ Dorothea Rjosk-Dendorfer $^{\mathrm{b}} \quad$ Anikó Sztrókay-Gaul $^{\mathrm{a}}$ Maximilian Reiser ${ }^{\mathrm{b}}$ \\ Susanne Grandla \\ ${ }^{a}$ Department of Breast Imaging, Red Cross Hospital, Munich, Germany; ${ }^{\mathrm{b}}$ Department of Radiology, \\ University Hospital, LMU, Munich, Germany
}

\section{Keywords}

Second-look ultrasound · Breast MRI · Breast cancer .

High-risk lesions

\begin{abstract}
Introduction: Due to the increasing use of dynamic breast MRI and the limited availability of MR-guided interventions, MRI-detected lesions usually undergo a second-look ultrasound (SLUS). We investigated the safety of a negative SLUS and a benign SLUS correlate in excluding malignant and high-risk lesions (B3) and evaluated criteria for the rate of detection on SLUS. Methods: In the retrospective analysis, all breast MRIs performed between 2011 and 2013 were screened for newly detected lesions. We analyzed the SLUS detection rate dependent on breast density, mass character, lesion size, and histology. We calculated the sensitivity, specificity, positive predictive value (PPV), and negative predictive value (NPV) of a negative and benign SLUS for malignant lesions (B5) and lesions requiring surgical excision (including high-risk and B5 lesions). Results: We successfully correlated 110 of 397 lesions. The detection rate was significantly higher for mass than for non-mass lesions and correlated with lesion size for mass lesions only. Lesions without/with a benign SLUS correlate were more frequently benign (including B3) or required no further procedure (B2). The sensitivity of SLUS in the detection of B3 and B5 lesions was $58 \%$, and $73 \%$ in the detection of B5 lesions. The NPV of a negative or benign SLUS for B3 and B5 lesions was 89\%,
\end{abstract}

and $96 \%$ for B5 lesions. Discussion: SLUS is a safe diagnostic tool for the management of MRI-detected lesions and can spare patients from undergoing invasive procedures.

(c) 2021 S. Karger AG, Basel

\section{Introduction}

Contrast-enhanced breast MRI represents a highly sensitive method, especially for women at a high risk for developing breast cancer [1]. In $16 \%$ of women with histologically proven breast cancer, preoperative MRI detects additional lesions and may contribute to a change in surgical procedure [2]. The technique was shown to have an excellent diagnostic performance in the assessment of noncalcified equivocal lesions [3], and to provide additional diagnostic information in some cases of mammographically detected microcalcifications [4]. However, the management of additional lesions detected on MRI poses a diagnostic challenge $[5,6]$.

MR-guided, vacuum-assisted biopsy of the breast is a safe and accurate sampling tool, but its use is limited by the low availability of MR devices and the high cost of the intervention [7-10]. Many MRI-detected lesions are therefore primarily evaluated by second-look ultrasound (SLUS). SLUS is a widely available, low-cost method for the localization and histological assessment of lesions first detected by contrast-enhanced breast MRI. Depending on lesion size and type, the reported negative predic- 
Table 1. MRI scanner and sequences used for diagnostic breast MRI

\begin{tabular}{lcllc}
\hline & $\begin{array}{l}\text { MAGNETOM } \\
\text { Verio (3-T) }\end{array}$ & $\begin{array}{l}\text { MAGNETOM }^{\circledR} \\
\text { Skyra (3-T) }\end{array}$ & $\begin{array}{l}\text { MAGNETOM }^{\circledR} \\
\text { Symphony }(1.5-T)\end{array}$ & $\begin{array}{l}\text { MAGNETOM }^{\circledR} \\
\text { Avanto (1.5-T) }\end{array}$ \\
\hline $\begin{array}{c}\text { T2-weighted } \\
\text { TR, ms }\end{array}$ & 5,230 & & & \\
TE, ms & 88 & 6,490 & 4,000 & 4,000 \\
FA, $^{\circ}$ & 127 & 107 & 71 & 67 \\
Matrix & $448 \times 425$ & $448 \times 448$ & $512 \times 512$ & $512 \times 307$ \\
\hline STIR & & & & \\
TR, ms & 6,700 & 770 & 4,920 & 4,920 \\
TE, ms & 55 & 57 & 68 & 66 \\
FA, ${ }^{\circ}$ & 137 & 162 & 180 & 180 \\
Matrix & $256 \times 230$ & $384 \times 268$ & $256 \times 256$ & $256 \times 256$ \\
\hline T1-weighted & & & & 9.1 \\
TR, ms & 6.5 & 5.23 & 9.1 & 4.76 \\
TE, ms & 2.47 & 2.46 & 4.76 & 25 \\
FA, ${ }^{\circ}$ & 15 & 10 & 25 & $256 \times 256$ \\
Matrix & $512 \times 512$ & $448 \times 425$ & $256 \times 256$ & \\
\hline
\end{tabular}

tive value (NPV) of SLUS is about $88 \%$ [11] and it is useful for downgrading MRI Breast Imaging Reporting and Data System (BI-RADS) IV lesions [12]. The detection rate is highest for malignant lesions and mass lesions [11, 13-19]. However, in some cases, achieving a correlation is a challenge due to the different positioning of the breast on MRI and ultrasound. No recommendations exist as to which MRI-detected lesions should undergo SLUS or how to ensure that the presumed SLUS correlate is identical to the MRI lesion [20,21]. Some lesions require MRguided biopsy or surgical excision after MR-guided wire localization. Which method is preferable depends on the size, type, and location of a lesion. Automated breast volumetric sonography [22] and virtual sonography [23] are promising methods for the assessment of MRI-detected lesions, but they are not widely available and are somewhat cost- and time-intensive.

This study was undertaken to evaluate the safety of SLUS as part of the diagnostic workup in patients after abnormal breast MRI in an academic breast center. To our knowledge, it is the first study to consider a negative SLUS (lesions without SLUS correlate) and a sonographically benign SLUS correlate (ultrasound BI-RADS II or III). To our knowledge, it is also the first study to evaluate the performance of SLUS separately for malignant lesions (histologically B5) and lesions needing total excision (B3 and B5). B3 lesions (high-risk lesions) including flat epithelial atypia, lobular neoplasia, papillary lesions, radial scars, atypical ductal hyperplasia, and phyllodes tumors have an overall risk for malignancy of $9.9-35.1 \%$, and should therefore be removed by vacuum-assisted biopsy or surgical excision $[24,25]$. High-risk lesions are diagnosed from percutaneous biopsy and the therapeutic procedure differs according to national guidelines [26, 27].

\section{Materials and Methods}

The reports of all breast MRI examinations performed between 1 January 2011 and 31 December 2013 were screened for lesions first reported from examination of dedicated breast MRI, and categorized as MR BI-RADS III, IV, or V. Indications for performing breast MRI were extracted from the medical report. All breast MRIs were retrospectively analyzed by 2 radiologists (in consensus). Lesion size, location, mass character, and breast density (according to the American College of Radiology [ACR] classification) were documented. The further workup of each lesion was assessed using the written reports. For each lesion, histopathological diagnosis, or follow-up (FU) of at least 24 months was used as the gold standard. If $>1$ histopathological diagnosis (i.e., core-needle biopsy and surgical excision) was available, the histology with the highest grading was used.

Breast MRI was performed with the patient in a prone position, with 1.5- and 3-tesla scanners and using a dedicated sensitivityencoding-enabled bilateral breast coil in the transversal slice direction. Scanners and scan protocols are listed in Table 1. In principle, the examination consisted of axial T2-weighted, short tau inversion recovery (STIR), nonenhanced, and contrast-enhanced T1weighted gradient-echo sequences after the manual injection of 0.2 $\mathrm{mL} / \mathrm{kg}$ meglumine gadopentetate (magnograf ${ }^{\circledR} 0.5 \mathrm{mmol} / \mathrm{mL}$ ) and subtraction images obtained after 2, 4, and $6 \mathrm{~min}$.

All SLUS examinations were performed using a high-resolution B-mode ultrasound system with a standard linear $(13.5 \mathrm{MHz})$ transducer (Siemens Acuson Antares, Siemens Healthcare, Germany). The diagnostic workup for each patient, that included the interpretation of initial imaging and the analysis of breast MRI and SLUS, was exclusively performed by 1 of 2 consultant radiologists with a minimum of 10 years' experience in breast diagnostics. The correlation between MRI and SLUS findings was performed according to a lesion's size, position within the breast parenchyma, and distance from other reproducible findings like cysts. For lesions undergoing biopsy, the compatibility of histology, MRI, and SLUS was thoroughly assessed. In cases of uncertain correlation between SLUS and MRI, MR-guided biopsy or short-term MRI FU was performed.

Statistical analysis was performed using SAS v9.4 for Windows (SAS Institute Inc., Cary, NC, USA). The positive predictive value 
Table 2. Histology of lesions for which SLUS was performed

\begin{tabular}{llll}
\hline Histology & $\begin{array}{l}\text { No SLUS } \\
\text { correlate, } n(\%)\end{array}$ & $\begin{array}{l}\text { Benign SLUS } \\
\text { correlate, } n(\%)\end{array}$ & $\begin{array}{l}\text { Suspicious SLUS correlate so } \\
\text { biopsy recommended, } n(\%)\end{array}$ \\
\hline $\begin{array}{l}\text { Benign } \\
\text { High-risk lesion }\end{array}$ & $56(19.5)$ & 0 & $28(36.8)$ \\
$\begin{array}{l}\text { Ductal carcinoma in situ } \\
\text { Invasive carcinoma }\end{array}$ & $9(3.9)$ & $1(2.8)$ & $10(13.2)$ \\
$\begin{array}{l}\text { Fibroadenoma } \\
\text { No histopathological assessment } \\
\text { follow-up only) }\end{array}$ & $17(0.3)$ & 0 & $6(7.9)$ \\
Total & $202(70.7)$ & 0 & $1(2.8)$ \\
\hline
\end{tabular}

(PPV) and NPV of SLUS were calculated separately for the differentiation of "lesions needing further invasive procedures" (invasive, noninvasive cancers, and B3 lesions) from "lesions needing no further procedure" (B2; Analysis A), and for the differentiation of malignant (invasive and noninvasive cancers) from benign lesions (B3 and B2; Analysis B). Lesions with a benign histology or unremarkable FU over 2 years were defined as "true benign."

For the calculation of sensitivity, specificity, PPV, and NPV, the variables "no equivalent" and "sonographically benign equivalent" (US BI-RADS II or III) were defined as "negative"; the variable "suspicious equivalent with indication to biopsy" was defined as "positive."

The correlation of detection rate with breast density, mass character, and histology, respectively, was calculated with a 2-sided Fisher's exact test. The correlation between detection rate and lesion size was calculated with Satterthwaite's test. $p<0.05$ was considered significant.

\section{Results}

Between January 2011 and December 2013, a total of 1,398 patients with 1,450 dedicated breast MRI examinations were eligible for the analysis. The mean age of all patients undergoing MRI was 50.0 (SD 12.3; range 13-85) years. Patients with no additional lesions on breast MRI were significantly older (mean 50.2; SD 12.5; range 13-85 years) than patients with $\geq 1$ additional lesions (mean 48.8; SD 11.9; range $21-81$ years) $(p=0.0346)$.

Indications for performing breast MRI were high-risk screening in $36.4 \%(n=528)$, FU after abnormal breast MRI in $31.0 \%(n=449)$, inconclusive conventional breast imaging in $24.9 \%(n=361)$, histologically proven breast cancer in $20.6 \%(n=298)$, clinical findings including pain or nipple discharge in $10.3 \%(n=149)$, detection of a palpable mass in $5.1 \%(n=74)$, and suspected recurrent disease in $4.4 \%(n=64)$. In some cases, $>1$ indication existed.

A total of 535 newly detected lesions were reported from 341 patient scans, 285 (53.3\%) located in the right breast and $250(46.7 \%)$ in the left breast. Most of the lesions $(n=149 ; 27.9 \%)$ were in the upper outer quadrant.
The distribution of breast density in patients with abnormal MRI was 1.2\% $(n=4)$ ACR A, $19.1 \%(n=65)$ ACR B, 51.9\% $(n=177)$ ACR C, and $19.6 \%(n=67)$ ACR D; $8.2 \%(n=28)$ had no mammography throughout the diagnostic workup.

The mean maximum diameter of all lesions on MRI was 11.6 (SD 9.0; range $2.0-70.0) \mathrm{mm} ; 53.5 \%(n=286)$ of these were non-mass lesions and $46 \%(n=249)$ were mass lesions.

SLUS was performed on 397 lesions. The remaining 138 lesions underwent MR-guided interventions or were resected together with the main tumor during mastectomy. Altogether, 110 of the 397 lesions (27.7\%) were successfully correlated on SLUS; 34 (30.1\% of correlated lesions) were classified as benign after SLUS (US BI-RADS II or III). After SLUS, biopsy was recommended for 76 lesions, and was performed on 67 lesions. A total of 287 lesions had no correlate on SLUS.

Table 2 gives an overview of the histology of lesions on which SLUS was performed. Please note that, for each lesion, either histology or FU (or both) was available.

Of the 535 newly MRI detected lesions (with and without SLUS), 170 (31.8\%) underwent either MRI- or ultrasound-guided breast biopsy. Only 103 (25.9\%) of the 397 lesions that underwent SLUS were biopsied. None of the lesions classified BI-RADS II or III after SLUS turned out to be malignant at FU or on histopathology. One woman without SLUS correlate who refused the recommended MRI-guided biopsy after SLUS presented with an invasive carcinoma during FU.

We analyzed the correlation of lesion detection rate in SLUS with the following parameters.

\section{Breast Density and Detection Rate}

$80.1 \%$ of the lesions without SLUS correlate versus $68.2 \%$ of the lesions with SLUS correlate were located in dense breasts (ACR C or D). In 100\% (2/2) of the lesions in ACR A breasts and 32.3\% (21/65) of those in ACR B breasts, a SLUS correlate was found. In $24.7 \%$ (53/215) of 

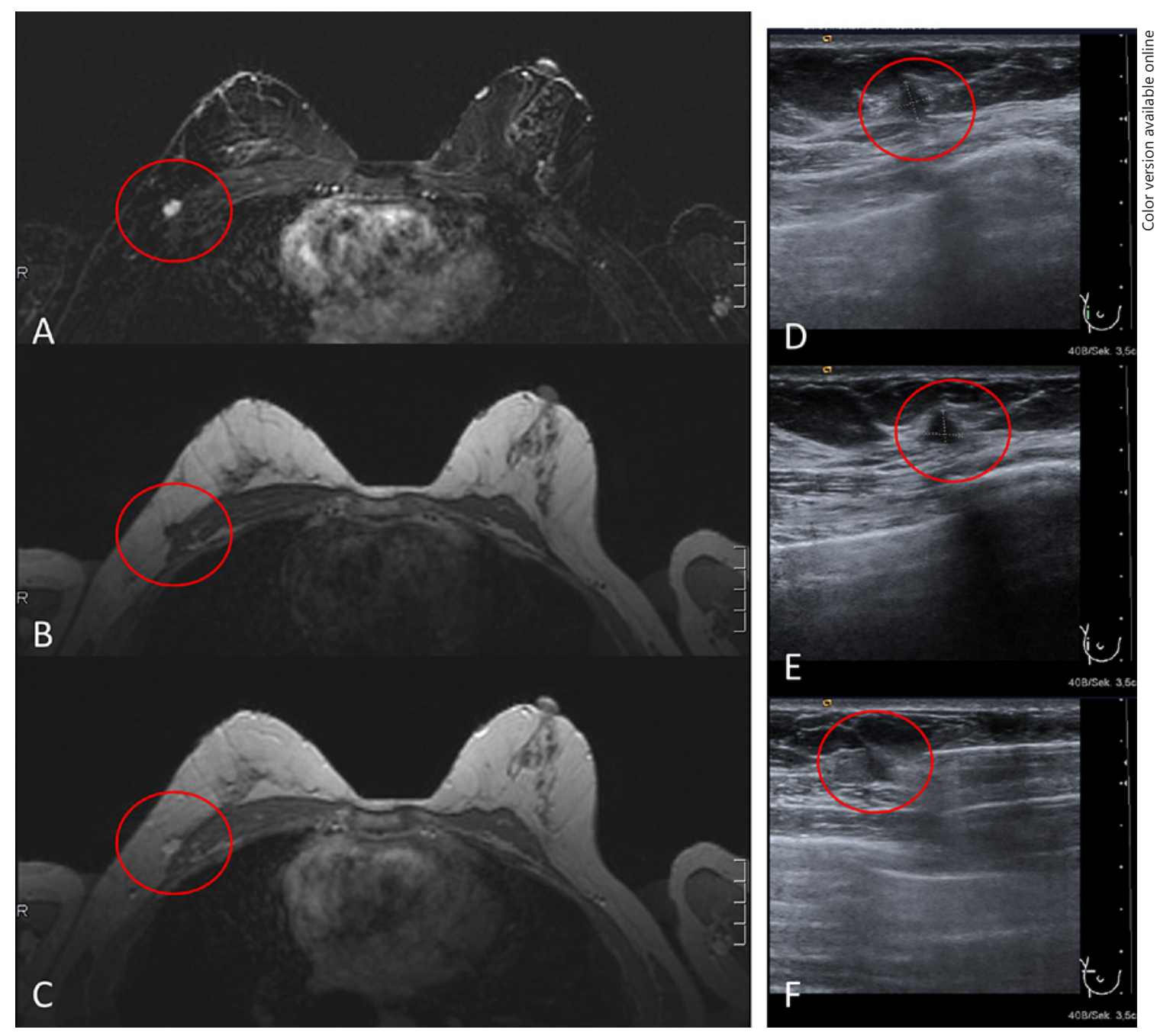

Fig. 1. Malignant lesion with SLUS correlate. Second subtraction image (A), native (B), and contrast-enhanced (C) T1-weighted images of a lesion with suspicious correlate on SLUS (D, E), that turned out to be an invasive carcinoma (no special type [NST]) on ultrasound-guided biopsy (F). BI-RADS V.

ACR C breasts and $24.4 \%(22 / 90)$ of ACR D breasts, a SLUS correlate was found. Lesions in ACR A and B breasts were more frequently correlated than lesions in ACR C and $\mathrm{D}$ breasts $(p=0.125)$.

\section{Mass Character and Detection Rate}

$50.6 \%(n=201)$ of the lesions on which SLUS was performed were non-mass lesions and $49.4 \%(n=196)$ were mass lesions, $38.3 \%(n=75)$ of the mass lesions versus $17.4 \%(n=35)$ of the non-mass lesions were successfully correlated, and $30.2 \%$ of the mass lesions and $15.3 \%$ of the non-mass lesions proved to be ductal carcinoma in situ (DCIS) or invasive cancer. The SLUS detection rate was significantly higher for mass lesions than for non-mass lesions $(p<0.001)$. Figure 1 shows a mass lesion with a suspicious SLUS correlate turning out to be an invasive carcinoma.

\section{Lesion Size and Detection Rate}

The mean maximum diameter of lesions without SLUS correlate (11.7 mm; 95\% CI 10.6-12.8 mm; SD $9.8 \mathrm{~mm}$ ) and of lesions with SLUS correlate $(11.6 \mathrm{~mm}$; $95 \%$ CI 10.4-12.9 mm; SD $6.6 \mathrm{~mm}$ ) was not significantly different. If only mass lesions are considered, the mean maximum diameter correlated significantly with the detectability ( $p=0.0257$ ). Mass lesions without SLUS correlate (9.0 mm; 95\% CI 7.8-10.3 mm; SD $7.2 \mathrm{~mm}$ ) were smaller in diameter than those with SLUS correlate $(11.0 \mathrm{~mm}$; 95\% CI 9.8-12.2 mm; SD $5.3 \mathrm{~mm}$ ). Figure 2 shows a mass lesion without SLUS correlate turning out to be a benign lesion.

The mean maximum diameter of non-mass lesions without and with SLUS correlate was $13.7 \mathrm{~mm}$ (95\% CI $12.0-15.4 \mathrm{~mm}$; SD $11.2 \mathrm{~mm}$ ) and $12.6 \mathrm{~mm}$ (95\% CI 9.8$15.5 \mathrm{~mm}$; SD $8.5 \mathrm{~mm}$ ), respectively. 


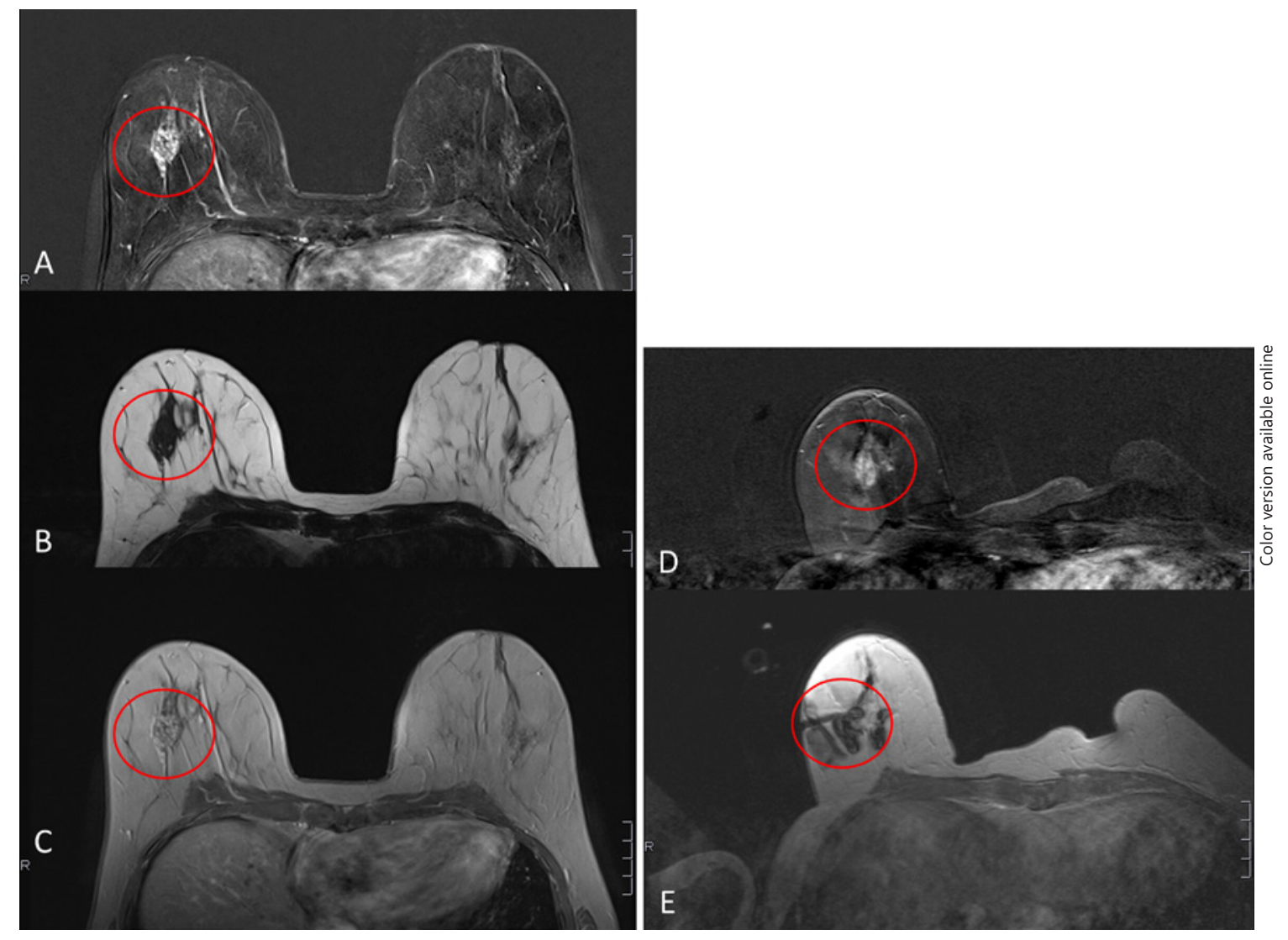

Fig. 2. Benign lesion without SLUS correlate. Oval mass lesion (red circle) in the lower outer quadrant of the right breast without SLUS correlate, histologically benign (fibroadenomatoid sclerosis, sclerosing adenosis, or apocrine epithelial metaplasy) on the second subtraction image (A), native (B), and contrast-enhanced (C) T1-weighted images. MR-guided wire localization including subtraction image (D) and postinterventional control (E) T1weighted image. MR BI-RADS IVb.

\section{Histology and Detection Rate}

The prevalence of invasive and noninvasive cancer within the SLUS collective (proven by ultrasound-guided or MRI-guided biopsy or surgical excision) was $9.3 \%$ $(n=37) .3 \cdot 5 \%(n=10)$ of the lesions (9 DCIS and 1 invasive cancer) without SLUS correlate versus $24.5 \%(n=27$; 6 DCIS and 21 invasive cancers) with SLUS correlate were malignant. The NPV of a negative or benign SLUS for invasive carcinoma was $98.9 \%$ of all sampled lesions.

Lesions without SLUS correlate were significantly more often B2 (Analysis A, $p=0.0187$ ) or benign (including B2 and B3; Analysis B, $p<0.0001$ ) than lesions with SLUS correlate.

Lesions without SLUS correlate or with a sonographically benign correlate were significantly more often $\mathrm{B} 2$ (Analysis A, $p=0.0193$ ) or benign (B2 or B3; $p<0.0001$ ) than lesions with a sonographically suspicious correlate.

\section{Sensitivity and Specificity of SLUS}

In Analysis A (detection of B3 and B5 lesions), the sensitivity of SLUS was $58.0 \%$ (95\% CI 45.5-69.8\%) and the specificity was $86.46 \%$ (95\% CI $81.8-90.1 \%)$. The PPV was 50.6 (95\% CI $39.1-62.1 \%)$ and the NPV was $89.5 \%$ (95\% CI 85.3-92.8\%).

In Analysis B (detection of B5 lesions), the sensitivity of SLUS was 73.2\% (95\% CI 57.1-85.8\%) and the specificity was $84.4 \%$ (95\% CI $79.9-88.2 \%$ ). The PPV was $38.0 \%$ (95\% CI 27.3-49.6\%) and the NPV was 96.0\% (95\% CI 930-98.0\%).

\section{Discussion}

Ultrasound is a widely available, low-cost method for the management of breast disease. It has evolved as an important tool in the assessment of lesions initially detected on breast MRI. However, there is no consensus on the specific indication for SLUS. The detection rates reported in the literature are highly heterogeneous, ranging from 23 to $82.1 \%$ [11].

At an academic breast center that specializes in highrisk screening, we retrospectively analyzed the accuracy of SLUS in the assessment of lesions initially identified on breast MRI. The focus of our study was to investigate if: 
1. There are imaging criteria that predict a successful correlation on SLUS.

2. SLUS can be helpful in the management of high-risk lesions.

3. A negative or benign SLUS finding can safely exclude malignancy, and hence justify a conservative approach with fine-mesh MRI and/or ultrasound control.

\section{Criteria Predicting Successful Correlation}

Mammographic breast density seems to influence detectability. Lesions in ACR A and B breasts were more often detected than lesions in ACR C and D breasts (not statistically significant). This is consistent with the findings of Bumberger et al. [28], who found that the detection rate was associated with the composition of the fibroglandular tissue determined on ultrasound. However, currently, the classification of mammographic and sonographic breast tissue composition is not standardized, leading to high interobserver variability $[29,30]$ which compromises the comparability of data.

The detection rate of mass lesions was significantly higher than that of non-mass lesions. This is consistent with previous studies $[11,28]$.

The literature is inconsistent about the correlation of lesion size and detectability. Whereas Spick and Baltzer [11] reported no effect of lesion size, Meissnitzer et al. [13], LaTrenta et al. [14], and Wiratkapun et al. [22] reported higher correlation rates for larger lesions than for smaller ones. This was most likely because non-mass lesions tend to be larger than mass lesions, although the detection of non-mass lesions on SLUS may be challenging. Our results indicated a correlation between lesion size and detection rate for mass lesions only.

The SLUS detection rate was correlated to the histology of a lesion. Lesions without a SLUS correlate were more frequently benign (B2 and B3) or required no further procedure (B2). Spick and Baltzer [11] reported a lesion detection rate of between 22.6 and $82.1 \%$ in their meta-analysis, higher for mass lesions than non-mass lesions and for malignant lesions than benign lesions, with a pooled PPV and NPV of 30.7 and $87.8 \%$, respectively.

\section{Management of High-Risk Lesions}

Since high-risk lesions are diagnostically challenging and of major interest in breast pathology, we placed particular emphasis on the detectability of these lesions. It remains a point of discussion whether high-risk lesions should be considered benign (since they formally are) or malignant (since they pose a certain risk of additional malignant findings and, depending on national guidelines, a complete resection is recommended) [26]. Spick and Baltzer [11] refer to the final histological diagnosis of these lesions. Since high-risk lesions (even if benign on final histology) are treated differently from B2 lesions di- agnosed from percutaneous biopsy, we performed 2 different analyses for this type of lesion.

\section{The Accuracy of SLUS}

Whereas previous studies define sensitivity, specificity, PPV, and NPV according to the detectability of a lesion, we integrated the interpretation made by the radiologist who performed the SLUS. We defined lesions without and with a sonographically benign correlate (USBI-RADS II or III) as "negative."

Abe et al. [18] and Laguna et al. [23] reported that classic malignant features on ultrasound were missing in a considerable percentage of initially MRI-detected lesions that turned out to be malignomas. Abe et al. [18] concluded that the threshold for ultrasound-guided biopsy should be lower than for lesions detected on standard ultrasound or mammography. Park et al. [21] recommend that the decision to perform biopsy should be based primarily on MRI criteria. Even though a classification of "benign" on SLUS is standardized by the BI-RADS catalogue, the subjective evaluation of the observer nevertheless plays an important role in the recommendation of further (invasive) procedures. In our investigation, 30.1\% of the correlated lesions were classified as benign after SLUS, and no malignancy was revealed on histology or at FU in any of these cases. This is represented by the excellent NPV of SLUS of $96 \%$ for invasive and noninvasive carcinoma. We conclude that a noninvasive approach is justified in cases with a benign SLUS correlate, provided that close controls with MRI and ultrasound will be performed. In any event, the indication for performing biopsy should consider the clinical context. From our investigation, $5.9 \%$ of the lesions without SLUS correlate turned out to be high-risk lesions, $3.1 \%$ as DCIS, and 1 $(0.3 \%)$ as invasive carcinoma. A missing SLUS correlate therefore did not exclude malignancy in our study.

The remarkably low detection rate of $27.7 \%$ needs further discussion and could be attributable to the following specific characteristics of our collective. On the one hand, the percentage of non-mass lesions (50.6\%) was high compared to that in other studies [29], and the detection rate of mass lesions was $38.3 \%$. On the other hand, we performed SLUS on $82.8 \%$ of MRI-detected lesions, i.e., most of the newly detected lesions underwent SLUS even if the probability of finding a correlate was rather low.

Most studies do not outline the percentage of SLUSdetected lesions compared to all MRI-detected lesions, thus limiting comparability. Moreover, we investigated a patient collective with a very low cancer prevalence of 9.3\% ( $n=37$ within the SLUS collective of 397 lesions) whereas Kolta et al. [12] reported a prevalence of $30.1 \%$. Altogether, $36.4 \%$ of the MRIs were performed for highrisk screening. The women who participate are usually premenopausal and often show hormone-related en- 
hancement. Moreover, this group of women undergoes MRI followed by ultrasound (formally SLUS) annually, so a broad indication of an A lesion detected on the MRI of a high-risk woman will consequently be described and referred to in the (immediately following) ultrasound examination even if it would usually be categorized as MRBI-RADS III. SLUS was able to reduce the biopsy rate from $31.8 \%$ (all MRI-detected lesions) to 25.9\% without missing any malignomas. We conclude that SLUS performed by a highly experienced professional can spare women from undergoing unnecessary interventions. This result is substantiated in a very recent work by Kolta et al. [12] and indicates that SLUS can downgrade MRIdetected lesions and thereby avoid unnecessary biopsies.

The probability of malignancy in lesions with SLUS correlate was higher than in lesions without SLUS correlate; this result is consistent with the literature [13-15, $18]$.

\section{Limitations}

Besides the retrospective design, a limitation of our study is that there were no standard criteria for when to perform SLUS. Nevertheless, SLUS was recommended for most of the lesions (82.8\%) initially detected on MRI. For most of the lesions, there was no final proof as to whether the estimated correlate really represented the MRI-detected lesion. This would require the placement of a marker inside the lesion followed by another MRI scan. In our institution, the examiner judges the compatibility and, if there is inconsistency, recommends further MRI-guided procedures.

\section{Conclusion}

SLUS is an accurate diagnostic tool for the management of MRI-detected lesions and has an excellent NPV, provided that the ultrasound is performed by an expert radiologist. Studies with standardized indication criteria for SLUS are desirable.

Not all lesions were histologically verified. However, the long FU period ( 24 months) could safely exclude malignancy.

\section{Statement of Ethics}

The study was conducted in accordance with the Declaration of Helsinki and was approved by the Institutional Review Board (Ethikkommission of the Ludwig-Maximilians-University, Munich, trial No. 573-16). Informed consent was waived by the Institutional Review Board.

\section{Conflict of Interest Statement}

The authors have no conflicts of interest to declare.

\section{Funding Sources}

Authors received no funding relevant to this study.

\section{Author Contributions}

K.H. contributed to the conception and data interpretation, was responsible for drafting the work, and agreed to ensure that questions related to the accuracy or integrity of any part of the work are appropriately investigated and resolved. H.D. contributed to the acquisition and analysis of data, and contributed to drafting the work. R.S. contributed to the statistical analysis of the data and critically revised the work. D.R.-D. and A.S.-G. contributed to the data acquisition and revised the work critically. M.R. contributed to the conception of the work and revised the work critically. S.G. contributed to the conception, data acquisition, analysis and interpretation, was responsible for drafting the work, and agreed to ensure that questions related to the accuracy or integrity of any part of the work are appropriately investigated and resolved. All authors approved the version to be published and agreed to be accountable for all aspects of the work.

\section{References}

1 Warner E, Messersmith H, Causer P, Eisen A, Shumak R, Plewes D. Systematic review: using magnetic resonance imaging to screen women at high risk for breast cancer. Ann Intern Med. 2008 May;148(9):671-9.

2 Houssami N, Ciatto S, Macaskill P, Lord SJ, Warren RM, Dixon JM, et al. Accuracy and surgical impact of magnetic resonance imaging in breast cancer staging: systematic review and meta-analysis in detection of multifocal and multicentric cancer. J Clin Oncol. 2008 Jul;26(19):3248-58.

3 Bennani-Baiti B, Bennani-Baiti N, Baltzer PA. Diagnostic Performance of Breast Magnetic Resonance Imaging in Non-Calcified Equivo- cal Breast Findings: Results from a Systematic Review and Meta-Analysis. PLoS One. 2016 Aug;11(8): e0160346.

4 Bennani-Baiti B, Baltzer PA. MR Imaging for Diagnosis of Malignancy in Mammographic Microcalcifications: A Systematic Review and Meta-Analysis. Radiology. 2017 Jun;283(3): 692-701.

5 Evans A, Trimboli RM, Athanasiou A, Balleyguier C, Baltzer PA, Bick U, et al.; European Society of Breast Imaging (EUSOBI), with language review by Europa Donna-The European Breast Cancer Coalition. Breast ultrasound: recommendations for information to women and referring physicians by the Eu- ropean Society of Breast Imaging. Insights Imaging. 2018 Aug;9(4):449-61.

6 Mann RM, Balleyguier C, Baltzer PA, Bick U, Colin C, Cornford E, et al.; European Society of Breast Imaging (EUSOBI), with language review by Europa Donna-The European Breast Cancer Coalition. Breast MRI: EUSOBI recommendations for women's information. Eur Radiol. 2015 Dec;25(12):3669-78.

7 Kuhl CK, Morakkabati N, Leutner CC, Schmiedel A, Wardelmann E, Schild HH. MR imaging-guided large-core (14-gauge) needle biopsy of small lesions visible at breast MR imaging alone. Radiology. 2001 Jul;220(1): $31-9$. 
8 Floery D, Helbich TH. MRI-Guided percutaneous biopsy of breast lesions: materials, techniques, success rates, and management in patients with suspected radiologic-pathologic mismatch. Magn Reson Imaging Clin N Am. 2006 Aug;14(3):411-25.

9 Perlet C, Heywang-Kobrunner SH, Heinig A Sittek H, Casselman J, Anderson I, et al. Magnetic resonance-guided, vacuum-assisted breast biopsy: results from a European multicenter study of 538 lesions. Cancer. 2006 Mar; 106(5):982-90.

10 Clauser P, Mann R, Athanasiou A, Prosch H, Pinker K, Dietzel M, et al. A survey by the European Society of Breast Imaging on the utilisation of breast MRI in clinical practice. Eur Radiol. 2018 May;28(5):1909-18.

11 Spick C, Baltzer PA. Diagnostic utility of second-look US for breast lesions identified at MR imaging: systematic review and metaanalysis. Radiology. 2014 Nov;273(2):401-9.

12 Kolta M, Clauser P, Kapetas P, Bernathova M, Pinker K, Helbich TH, et al. Can second-look ultrasound downgrade MRI-detected lesions? A retrospective study. Eur J Radiol. 2020 Jun; 127:108976.

13 Meissnitzer M, Dershaw DD, Lee CH, Morris EA. Targeted ultrasound of the breast in women with abnormal MRI findings for whom biopsy has been recommended. AJR Am J Roentgenol. 2009 Oct;193(4):1025-9.

14 LaTrenta LR, Menell JH, Morris EA, Abramson AF, Dershaw DD, Liberman L. Breast lesions detected with MR imaging: utility and histopathologic importance of identification with US. Radiology. 2003 Jun;227(3): 856-61.

15 Demartini WB, Eby PR, Peacock S, Lehman CD. Utility of targeted sonography for breast lesions that were suspicious on MRI. AJR Am J Roentgenol. 2009 Apr;192(4):1128-34.

16 Linda A, Zuiani C, Londero V, Bazzocchi M. Outcome of initially only magnetic resonance mammography-detected findings with and without correlate at second-look sonography: distribution according to patient history of breast cancer and lesion size. Breast. 2008 Feb; 17(1):51-7.

17 Sim LS, Hendriks JH, Bult P, Fook-Chong SM. US correlation for MRI-detected breast lesions in women with familial risk of breast cancer. Clin Radiol. 2005 Jul;60(7):801-6.

18 Abe H, Schmidt RA, Shah RN, Shimauchi A, Kulkarni K, Sennett CA, et al. MR-directed ("Second-Look") ultrasound examination for breast lesions detected initially on MRI: MR and sonographic findings. AJR Am J Roentgenol. 2010 Feb;194(2):370-7.

19 Candelaria R, Fornage BD. Second-look US examination of MR-detected breast lesions. J Clin Ultrasound. 2011 Mar-Apr;39(3):115-21.

20 Monticciolo DL. Postbiopsy confirmation of MR-detected lesions biopsied using ultrasound. AJR Am J Roentgenol. 2012 Jun; 198(6):W618-20.

21 Park VY, Kim MJ, Kim EK, Moon HJ. Second-look US: how to find breast lesions with a suspicious MR imaging appearance. Radiographics. 2013 Sep-Oct;33(5):1361-75.

22 Wiratkapun C, Duke D, Nordmann AS, Lertsithichai P, Narra V, Barton PT, et al. Indeterminate or suspicious breast lesions detected initially with MR imaging: value of MRI-directed breast ultrasound. Acad Radiol. 2008 May;15(5):618-25.

23 Laguna AD, Arranz SJ, Checa VQ, Roca SA, Jiménez DE, Oliver-Goldaracena J. Sonographic findings of additional malignant le- sions in breast carcinoma seen by second look ultrasound. J Clin Imaging Sci. 2011;1:34.

24 Rageth CJ, O’Flynn EA, Pinker K, KubikHuch RA, Mundinger A, Decker T, et al. Second International Consensus Conference on lesions of uncertain malignant potential in the breast (B3 lesions). Breast Cancer Res Treat. 2019 Apr;174(2):279-96.

25 Bianchi S, Caini S, Renne G, Cassano E, Ambrogetti D, Cattani MG, et al.; VANCB Study Group. Positive predictive value for malignancy on surgical excision of breast lesions of uncertain malignant potential (B3) diagnosed by stereotactic vacuum-assisted needle core biopsy (VANCB): a large multi-institutional study in Italy. Breast. 2011 Jun;20(3):264-70.

26 Ferré R, Kao E, Mesurolle B. High-Risk Lesions Detected at Second-Look US for Breast Lesions Identified at MR Imaging. Radiology. 2015 Aug;276(2):614-6.

27 Spick C, Baltzer PA. Response. Radiology. 2015 Aug;276(2):615-6.

28 Bumberger A, Clauser P, Kolta M, Kapetas P, Bernathova M, Helbich TH, et al. Can we predict lesion detection rates in second-look ultrasound of MRI-detected breast lesions? A systematic analysis. Eur J Radiol. 2019 Apr; 113:96-100.

29 Sartor H, Lång K, Rosso A, Borgquist S, Zackrisson S, Timberg P. Measuring mammographic density: comparing a fully automated volumetric assessment versus European radiologists' qualitative classification. Eur Radiol. 2016 Dec;26(12):4354-60.

30 Kim WH, Lee SH, Chang JM, Cho N, Moon WK. Background echotexture classification in breast ultrasound: inter-observer agreement study. Acta Radiol. 2017 Dec;58(12): 1427-33. 